\title{
Sliding-mode observers based distributed consensus control for nonlinear multi-agent systems with disturbances
}

\author{
Yulian Jiang ${ }^{1} \cdot$ Yuhang Zhang ${ }^{1} \cdot$ Hongquan Wang $^{2} \cdot$ Keping Liu $^{1}$ (])
}

Received: 8 October 2020 / Accepted: 9 March 2021 / Published online: 10 April 2021

(c) The Author(s) 2021

\begin{abstract}
The distributed consensus control problem for nonlinear multi-agent systems (MASs) with external disturbances under switching directed topologies is investigated. Distributed sliding-mode observers are designed considering both nonlinear dynamics and disturbances in MASs. Utilizing estimated states information via sliding-mode observers, a control protocol is constructed and analyzed to ensure the MASs reach consensus, and additionally guarantee the desired disturbance rejection criterion. Furthermore, the simulation experiment is carried out by taking multiple simple-pendulum network systems. By comparing this work with the related existing results, our designed observers are superior in estimating states information simultaneously considering both nonlinear dynamics and external disturbances, and the experiment result analysis shows validity of distributed consensus algorithm based on sliding-mode observers for MASs.
\end{abstract}

Keywords Multi-agent systems $\cdot$ Sliding-mode observer $\cdot$ Nonlinear dynamics $\cdot$ Disturbance $\cdot$ Consensus

\section{Introduction}

In recent years, as a significant research branch of distributed artificial intelligence (DAI) and computational intelligence, the distributed cooperative control for multi-agent systems (MASs) has become a hot research topic. The related applications of DAI include mobile robots [1], vehicle fleet [2], satellite formation [3], multiple biomimetic robotic fish [4], unmanned aerial vehicle [5], unmanned ground vehicle [6], and so on. Through information exchanges, a group of agents can greatly enhance the intelligence degree of individual behavior and work cooperatively to accomplish some complex tasks that can not be successfully done by a single agent [7-23]. Wen et al. [7] studied the consensus controller of MASs with general linear dynamics and a fixed directed topology. By constructing a reduced-order observer-based protocol, Li et al. [8] devoted to the consensus problem for linear MASs under directed communication topologies. Wang et al. [9] researched the distributed tracking consensus

Keping Liu

liukeping@ccut.edu.cn

1 Department of Electrical and Electronic Engineering, Changchun University of Technology, Changchun, China

2 Wulanchabu Electric Power Bureau, Inner Mongolia Electric Power (Group) Co., Ltd, Wulanchabu, China problem for nonlinear leader-following MASs. Zou et al. [11] studied the adaptive output-feedback stabilization problem of stochastic strict-feedback systems with sensor uncertainty and unknown growth rate and stochastic disturbance. Liu et al. [12-14] mainly devoted to resolve three types of fixedtime event-triggered consensus problems for MASs.

From above literatures, we can see that the control protocol based on state feedback can resolve the consensus problem effectively, with the premise, is that the neighboring agents' states are available. However, in practice, the states of MASs are always unavailable and only the output can be measured as feedback information [17-20]. Thus, it is necessary to design a suitable observer to estimate system states actually. [24] proposed a fuzzy observer for the periodic tracking control problem of nonlinear systems. In addition, a sliding-mode observer also can be devised to estimate the unavailable state. Sliding-mode observers not only provide practical possibilities for the technical implementation of state feedback, but also have been applied in many aspects of control engineering, e.g. replicating perturbation for achieving complete compensation to the disturbance and so on. Furthermore, in sliding-mode observers, the sliding mode can be designed artificially in advance, and its motion equation only depends on the selected switching function, while it is independent of the system parameters and external disturbances. Therefore, it possesses strong robustness. In [25,26], 
sliding-mode observers with good robustness were designed for uncertain systems. For second-order nonlinear MASs, [27] studied consensus problems via sliding mode observer and controller, and for nonlinear MASs with high-order integral chained differentiators, the distributed adaptive fuzzy control via sliding-mode observers was proposed in [28]. In [29] and [30], sliding-observers based output consensus control were designed for homogeneous multi-agent systems and heterogeneous multi-agent systems, respectively. The observer-based output feedback control problem of network physical systems with periodic denial of service (DoS) attacks was studied in [31]. In most of the above existing literatures, sliding-mode observers were always designed without considering either external disturbances or nonlinear dynamics for general MASs, which motivates us to do the further work.

This work studies consensus control for MASs considering both nonlinearity and unknown disturbances via observer-based dynamic output method under directed switching topologies. The main contributions of our work are summarized as follows: (1) To estimate the actual and unavailable states, a local sliding-mode observer is constructed for nonlinear MASs with disturbances. (2) A novel distributed consensus controller under switching topologies is designed, in which some parameters are computed via the inequality. (3) Utilizing the Lyapunov function and LMI technique, sufficient conditions for MASs, the controller gain and observer gain matrices are obtained simultaneously. (4) The result presented in this work can resolve the consensus for nonlinear MASs with unavailable states and disturbances under directed switching topologies. Comparing with literatures [12-14], we design a type of distributed consensus control protocol based on constructed sliding-mode observers with considering unavailable states, nonlinear dynamics and unknown disturbances, simultaneously. Our work is different from $[25,26]$, where the observers were designed for traditional single-systems, the sliding-mode observers in our results could estimate states for MASs as one problem of DAI. Compared to [19] which investigated consensus for linear MASs via reduced-order observers, our theoretical approach is able to solve the disturbances and nonlinear dynamics. In contrast to the consensus protocol proposed in [32] which imposed the constraint that the state information is available, our controller based on sliding-mode observers could resolve the unavailable states, as well as disturbances and nonlinear dynamics.

\section{Graph theory and problem formulation}

\section{Graph theory}

The interaction topologies between agents is described by the directed graph $G=(v, \varepsilon, A)$, in which $v=\left\{v_{1}, \ldots, v_{n}\right\}$ denotes the set of nodes, $\varepsilon \subset v \times v$ the set of edges and $A=$ $\left[a_{i j}\right]$ the adjacency matrix. If $(i, j) \in \varepsilon$, then $a_{i j}=1$, otherwise $a_{i j}=0$. In particular, $a_{i i}=0$. The Laplacian matrix for graph $G$ is $L=D-A$, with $D=\operatorname{diag}\left\{d_{1}, \ldots, d_{n}\right\}$ is called its degree matrix whose diagonal element of the matrix $D$ is $d_{i}=\sum_{j=1}^{n} a_{i j}, i=1, \ldots, n$.

Here a piecewise constant switching signal function $\varsigma(t)$ : $[0, \infty) \mapsto\{1,2, \ldots, m\} \triangleq \Omega$ represents $m$ variable topologies. Let $\lambda_{\varsigma(t) i}$ be the ith positive real eigenvalue of Laplacian matrix $L_{\varsigma(t)}$ corresponding to graph $G_{\varsigma(t)}$, in which $\lambda_{\max }$ and $\lambda_{\min }$ are the maximum and minimum nonzero eigenvalues, respectively. Moreover, we assume $G_{\zeta(t)}$ is directed and balanced in this work.

Lemma 1 [33] Define symmetric matrix $L_{c}=\left[L_{c_{i j}}\right] \in$ $R^{n \times n}$, where $L_{c_{i j}}=\left\{\begin{array}{ll}(n-1) / n, & i=j \\ -1 / n, & i \neq j\end{array}\right.$, and some properties are as follows:

1. The eigenvalues of $L_{c}$ include 1 with multiplicity $n-$ 1 and 0 with multiplicity $1 . \mathbf{1}_{n}^{T}$ and $\mathbf{1}_{n}$ are respectively the left and right eigenvectors of $L_{c}$ related to the zero eigenvalue.

2. There is an orthogonal matrix $U$, with $\mathbf{1}_{n} / \sqrt{n}$ as the last column, and thus satisfying $U^{T} L_{c} U=\left[\begin{array}{cc}I_{n-1} & 0 \\ 0 & 0\end{array}\right]$. Let $L \in R^{n \times n}$ be the Laplacian matrix of any balanced graph, and hence $U^{T} L U=\left[\begin{array}{cc}L_{1} & 0 \\ 0 & 0\end{array}\right]$, with the positive definite matrix $L_{1} \in R^{(n-1) \times(n-1)}$ in the connected graph. Hence, there is an orthogonal matrix $\Pi_{1}$, which makes $L_{1}=\Pi_{1} \Lambda_{1} \Pi_{1}^{T}$, with $\Lambda_{1}=\operatorname{diag}\left\{\lambda_{1} \ldots, \lambda_{n-1}\right\}$, in which $\lambda_{1} \ldots, \lambda_{n-1}$ are $n-1$ eigenvalues of Laplacian matrix.

\section{Problem formulation}

Consider the nonlinear MASs with the dynamic model as

$$
\left\{\begin{array}{cc}
\dot{x}_{i}(t)=A x_{i}(t)+B u_{i}(t)+f\left(x_{i}(t)\right)+D \omega_{i}(t) \\
y_{i}(t)=C x_{i}(t), & i=1,2, \ldots, n
\end{array}\right.
$$

in which $x_{i}(t) \in R^{r}, u_{i}(t) \in \mathrm{R}^{m}$ and $y_{i}(t) \in \mathrm{R}^{p}$ are respectively the state, control input (control protocol) and measurement output. $f\left(x_{i}(t)\right)$ is a nonlinear function and $\omega_{i}(t) \in L_{2}(0, \infty)$ is disturbance. $A, B, C, D$ are constant 
matrices with the appropriate dimensions. $B$ and $C$ are fullcolumn rank and full-row rank matrices, respectively.

The controlled output function is chosen as

$z_{i}(t)=x_{i}(t)-\frac{1}{n} \sum_{j=1}^{n} x_{j}(t), \quad i=1, \ldots n$,

Definition 1 For a class of MASs (1), if all the states of agents satisfy

$\lim _{t \rightarrow \infty}\left(x_{i}(t)-x_{j}(t)\right)=0, \forall i, j \in\{1,2, \ldots, n\}$

the MASs (1) is asymptotically convergent, and reach state consensus. And the control target is to construct the consensus controller $u_{i}(t)$ such that

$\int_{0}^{\infty}\|z(t)\|^{2} \mathrm{~d} t<\gamma^{2} \int_{0}^{\infty}\|\bar{\omega}(t)\|^{2} \mathrm{~d} t$

where $z(t)=\left(z_{1}^{T}(t), \ldots, z_{n}^{T}(t)\right)^{T}, \omega(t)=\left(\omega_{1}^{T}(t), \ldots\right.$, $\left.\omega_{n}^{T}(t)\right)^{T}, \bar{\omega}_{i}(t)=\omega_{i}(t)-\frac{1}{n} \sum_{j=1}^{n} \omega_{j}(t), \bar{\omega}(t)=\left(\bar{\omega}_{1}^{T}(t)\right.$, $\left.\ldots, \bar{\omega}_{n}^{T}(t)\right)^{T}, \forall \bar{\omega}(t) \in L_{2}[0, \infty)$, and $\gamma>0$ is a given $H_{\infty}$ performance index.

Assumption 1 The nonlinear function $f\left(x_{i}(t)\right)$ satisfies the Lipschitz condition with a Lispschitz constant $\mu>0$, that is

$\|f(\mathrm{x})-f(y)\| \leq \mu\|x-y\|, \forall x, y \in R^{r}$

\section{Graph theory and problem formulation}

For system (1), we propose the following sliding-mode observers

$\dot{\hat{x}}_{i}(t)=A \hat{x}_{i}(t)+B u_{i}(t)+G\left(\hat{y}_{i}(t)-y_{i}(t)\right)+f\left(\hat{x}_{i}(t)\right)+D v_{i}(t)$

where $\hat{x}_{i}(t)$ is the estimated state, and $v_{i}(t) \in R$ is the nonlinear input (sliding-mode strategy) of the observer. $G$ is the gain matrix. $D$ is a constant matrix with the appropriate dimensions.

Set state error for the ith agent as

$e_{i}(t)=\hat{x}_{i}(t)-x_{i}(t)$

and in this work, the nonlinear input is designed as

$v_{i}(t)=\left\{\begin{array}{cc}-\rho \frac{F \Psi_{i}(t)}{\left\|F \Psi_{i}(t)\right\|}, & \Psi_{i}(t) \neq 0 \\ 0, & \Psi_{i}(t)=0\end{array}\right.$

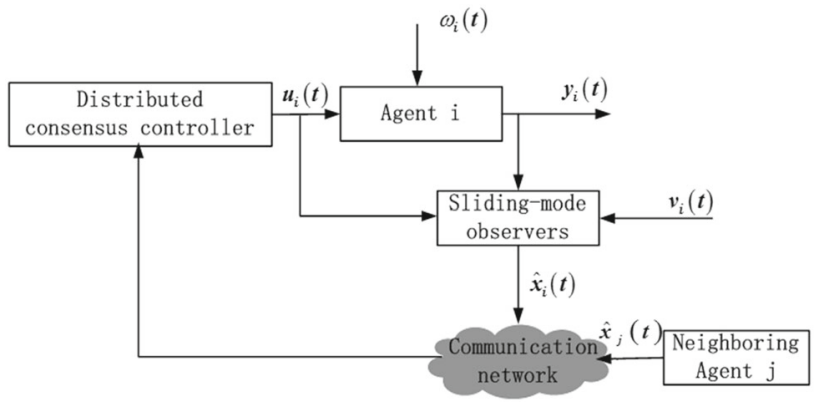

Fig. 1 Distributed consensus control structure

where $\Psi_{i}(t)=C \hat{x}_{i}(t)-y_{i}(t), F$ matrix to be solved, and $\rho$ is a given positive constant. From (3) and (4), we have

$$
\begin{aligned}
\dot{e}_{i}(t)= & (A+G C) e_{i}(t)+f\left(\hat{x}_{i}(t)\right)-f\left(x_{i}(t)\right) \\
& +D\left(v_{i}(t)-\omega_{i}(t)\right)
\end{aligned}
$$

Set $e(t)=\left(e_{1}^{T}(t), \ldots, e_{n}^{T}(t)\right)^{T}, v(t)=\left(v_{1}^{T}(t), \ldots\right.$, $\left.v_{n}^{T}(t)\right)^{T}$ and (6) is further rewritten as

$\dot{e}(t)=\left[I_{n} \otimes(A+G C)\right] e(t)+\left[\begin{array}{c}f\left(\hat{x}_{1}(t)\right)-f\left(x_{1}(t)\right) \\ \vdots \\ f\left(\hat{x}_{n}(t)\right)-f\left(x_{n}(t)\right)\end{array}\right]$

\section{Consensus control protocol design based on estimated system states}

According to Definition 1, the following consensus control protocol is constructed

$u_{i}(t)=K \sum_{j \in N_{i}(t)} a_{i j}(t)\left(\hat{x}_{i}(t)-\hat{x}_{j}(t)\right)$

where $K \in \mathrm{R}^{m \times r}$ represents the control gain matrix.

The structure for the proposed distributed consensus control (8) based on sliding-mode observers (3) of MASs (1) is shown in Fig. 1. It is the distributed consensus control structure based on sliding-mode observers (3) of MASs (1), which is composed of agents, communication network, a slidingmode observer and a distributed consensus controller. The main concern of this work is how to design and analyze the distributed consensus controller based on sliding-mode observers under directed switching topologies in networks.

According to Lemma 1, we have

$$
\begin{aligned}
& u(t)=\left(L_{\zeta(t)} \otimes K\right) \hat{x}(t) \\
& z(t)=\left(L_{c} \otimes I_{r}\right) x(t)
\end{aligned}
$$


with $u(t)=\left(u_{1}^{T}(t), \ldots, u_{n}^{T}(t)\right)^{T}, x(t)=\left(x_{1}^{T}(t), \ldots\right.$, $\left.x_{n}^{T}(t)\right)^{T}, \hat{x}(t)=\left(\hat{x}_{1}^{T}(t), \ldots, \hat{x}_{n}^{T}(t)\right)^{T}$.

Based on (2), the derivative of controlled output $z_{i}(t)$ is

$$
\begin{aligned}
\dot{z}_{i}(t)= & A\left(x_{i}(t)-\frac{1}{n} \sum_{j=1}^{n} x_{j}(t)\right)+B\left(u_{i}(t)-\frac{1}{n} \sum_{j=1}^{n} u_{j}(t)\right) \\
& +f\left(x_{i}(t)\right)-\frac{1}{n} \sum_{j=1}^{n} f\left(x_{j}(t)\right)+D \bar{\omega}_{i}(t)
\end{aligned}
$$

The following main result is provided.

Theorem 1 For MASs (1), we set $P_{2} D=C^{T} F^{T},\left\|\omega_{i}(t)\right\| \leq$ $\rho$. If there exists positive definite matrices $P_{1}, P_{2}$, positive constants $\mu, \gamma$ and matrices $Q_{1}, Q_{2}$, the inequality

$$
\left[\begin{array}{ccccc}
\Omega & P_{1} D & \lambda_{\zeta(t) i} Q_{1} & 0 & P_{1} \\
* & -\gamma^{2} I & 0 & 0 & 0 \\
* & * & M & P_{2} & 0 \\
* & * & * & -\frac{1}{\mu} I & 0 \\
* & * & * & * & -\frac{1}{\mu} I
\end{array}\right]<0
$$

is satisfied, where $\Omega=P_{1} A+A^{T} P_{1}+\lambda_{\varsigma(t) i} Q_{1}+\lambda_{5(t) i} Q_{1}^{T}+$ $(\mu+1) I, M=P_{2} A+A^{T} P_{2}+Q_{2}+Q_{2}^{T}+\mu I, Q_{1}=$ $P_{1} B K, Q_{2}=P_{2} G C, P_{2} D=C^{T} F^{T}, \lambda_{S(t) i}=\lambda_{\min }$ and $\lambda_{\max } \cdot \mu>0$ denotes the Lispschitz constant. The system (1) is asymptotically stable, with $H_{\infty}$ performance index $\gamma$ satisfying Definition 1.

Proof Construct Lyapunov function

$V(t)=z^{T}(t)\left(I_{n} \otimes P_{1}\right) z(t)+e^{T}(t)\left(I_{n} \otimes P_{2}\right) e(t)$

According to (10), (11) and Lemma 1, compute the derivative of $V(t)$ as

$$
\begin{aligned}
\dot{V}(t)= & 2 z^{T}(t)\left(I_{n} \otimes P_{1}\right) \dot{z}(t)+2 e^{T}(t)\left(I_{n} \otimes P_{2}\right) \dot{e}(t) \\
= & 2 z^{T}(t)\left(L_{c} \otimes P_{1} A\right) x(t)+2 z^{T}(t)\left(L_{c} \otimes P_{1} B\right) u(t) \\
& +2 \sum_{i=1}^{n} z_{i}^{T}(t) P_{1}\left[f\left(x_{i}(t)\right)-\frac{1}{n} \sum_{j=1}^{n} f\left(x_{j}(t)\right)\right] \\
& +2 z^{T}(t)\left(I_{n} \otimes P_{1} D\right) \bar{\omega}(t) \\
& +2 e^{T}(t)\left[I_{n} \otimes P_{2}(A+G C)\right] e(t) \\
& +2 \sum_{i=1}^{n} e_{i}^{T}(t) P_{2}\left[f\left(\hat{x}_{i}(t)\right)-f\left(x_{i}(t)\right)\right] \\
& +2 e^{T}(t)\left(I_{n} \otimes P_{2} D\right)(v(t)-\omega(t))
\end{aligned}
$$

Here, we set $\bar{x}(t)=\frac{1}{n} \sum_{j=1}^{n} x_{j}(t)$. Because $\sum_{i=1}^{n} z_{i}(t)=0$, ones obtain

$$
\begin{aligned}
& 2 \sum_{i=1}^{n} z_{i}^{T}(t) P_{1}\left[f\left(x_{i}(t)\right)-\frac{1}{n} \sum_{j=1}^{n} f\left(x_{j}(t)\right)\right] \\
& =2 \sum_{i=1}^{n} z_{i}^{T}(t) P_{1}\left[f\left(x_{i}(t)\right)-f(\bar{x}(t))\right] \\
& \quad+2\left(\sum_{i=1}^{N} z_{i}^{T}(t)\right) P_{1}\left[f(\bar{x}(t))-\frac{1}{n} \sum_{j=1}^{n} f\left(x_{j}(t)\right)\right] \\
& =2 \sum_{i=1}^{n} z_{i}^{T}(t) P_{1}\left[f\left(x_{i}(t)\right)-f(\bar{x}(t))\right]
\end{aligned}
$$

According to Assumption 1, (15) can be transformed as

$$
\begin{aligned}
& 2 \sum_{i=1}^{n} z_{i}^{T}(t) P_{1}\left[f\left(x_{i}(t)\right)-\frac{1}{n} \sum_{j=1}^{n} f\left(x_{j}(t)\right)\right] \\
& \quad \leq 2 \mu \sum_{i=1}^{n}\left\|z_{i}^{T}(t) P_{1}\right\|\left\|\left(x_{i}(t)-\frac{1}{n} \sum_{j=1}^{n} x_{j}(t)\right)\right\| \\
& \quad=2 \mu \sum_{i=1}^{n}\left\|z_{i}^{T}(t) P_{1}\right\|\left\|z_{i}(t)\right\|
\end{aligned}
$$

Set $Q_{2}=P_{2} G C$, and based on Assumption 1, then combining (9),(10) and (14), we can get

$$
\begin{aligned}
\dot{V}_{1}(t) \leq & 2 x^{T}(t)\left(L_{c}^{T} L_{c} \otimes P_{1} A+L_{c}^{T} L_{c} L_{\zeta}(t) \otimes P_{1} B K\right) x(t) \\
& +2 x^{T}(t)\left(L_{c}^{T} L_{c} L_{\zeta(t)} \otimes P_{1} B K\right) e(t) \\
& +x^{T}(t)\left(L_{c}^{T} L_{c} \otimes\left(\mu P_{1}^{2}+\mu I\right)\right) x(t) \\
& +2 x^{T}(t)\left(L_{c}^{T} \otimes P_{1} D\right) \bar{\omega}(t) \\
& +\mathrm{e}^{T}(t)\left(I_{N} \otimes\left(P_{2} A+A^{T} P_{2}+Q_{2}+Q_{2}^{T}\right)\right) e(t) \\
& +\mathrm{e}^{T}(t)\left(I_{N} \otimes\left(\mu I+\mu P_{2} P_{2}\right)\right) e(t) \\
& +2 \sum_{i=1}^{N} e_{i}^{T}(t) P_{2} D\left(v_{i}(t)-\omega_{i}(t)\right)
\end{aligned}
$$

Combining (5), $P_{2} D=C^{T} F^{T}$ and $\left\|\omega_{i}(t)\right\| \leq \rho$, we have

$$
\begin{aligned}
& 2 \sum_{i=1}^{n} e_{i}^{T}(t) P_{2} D\left(v_{i}(t)-\omega_{i}(t)\right) \\
& \quad=2 \sum_{i=1}^{n} e_{i}^{T}(t) P_{2} D\left(-\rho \frac{F\left(C \hat{x}_{i}(t)-y_{i}(t)\right)}{\left\|F\left(C \hat{x}_{i}(t)-y_{i}(t)\right)\right\|}-\omega_{i}(t)\right)
\end{aligned}
$$




$$
\begin{aligned}
& \leq 2 \sum_{i=1}^{n}\left(-\rho \cdot\left\|e_{i}^{T}(t) P_{2} D\right\|+\left\|e_{i}^{T}(t) P_{2} D\right\| \cdot\left\|\omega_{i}(t)\right\|\right) \\
& \leq 2 \sum_{i=1}^{n}\left\|e_{i}^{T}(t) P_{2} D\right\| \cdot(-\rho+\rho) \\
& =0
\end{aligned}
$$

So, we can obtain

$$
\begin{aligned}
\dot{V}(t) \leq & 2 x^{T}(t)\left(L_{c}^{T} L_{c} \otimes P_{1} A+L_{c}^{T} L_{c} L_{\zeta(t)} \otimes P_{1} B K\right) x(t) \\
& +2 x^{T}(t)\left(L_{c}^{T} L_{c} L_{\zeta(t)} \otimes P_{1} B K\right) e(t) \\
& +x^{T}(t)\left(L_{c}^{T} L_{c} \otimes\left(\mu_{1} P_{1}^{2}+\mu I\right)\right) x(t) \\
& +e^{T}(t)\left(I_{n} \otimes\left(P_{2} A+A^{T} P_{2}+Q_{2}+Q_{2}^{T}+\mu I+\mu P_{2}^{2}\right)\right) e(t) \\
& +2 x^{T}(t)\left(L_{c}^{T} \otimes P_{1} D\right) \bar{\omega}(t)
\end{aligned}
$$

Based on Lemma 2, ones have

$$
\begin{aligned}
& U_{\zeta(t)}^{T} L_{c} U_{\zeta(t)}=\left[\begin{array}{cc}
I_{n-1} & 0 \\
0 & 0
\end{array}\right] \triangleq I^{\prime}{ }_{n} \\
& U_{\zeta(t)}^{T} L_{\zeta(t)} U_{\zeta(t)}=\left[\begin{array}{cc}
L_{1} & 0 \\
0 & 0
\end{array}\right] \triangleq L^{\prime}
\end{aligned}
$$

in which, $U_{\zeta(t)} \in R^{n}$ is an orthogonal matrix, and $L_{1}>0$ since the balanced and directed graph $G_{\zeta(t)}$ is connected. Then we can define:

$$
\left\{\begin{array}{l}
\varphi_{\zeta(t)}(t)=\left(\varphi_{\zeta(t) 1}^{T}(t), \ldots, \varphi_{\zeta(t) n}^{T}(t)\right)^{T}=\left(U_{\zeta(t)}^{T} \otimes I_{r}\right) x(t) \\
\sigma_{\zeta(t)}(t)=\left(\sigma_{\zeta(t) 1}^{T}(t), \ldots, \sigma_{\zeta(t) n}^{T}(t)\right)^{T}=\left(U_{\zeta(t)}^{T} \otimes I_{r}\right) \bar{\omega}(t) \\
\chi_{\zeta(t)}(t)=\left(\chi_{\zeta(t) 1}^{T}(t), \ldots, \chi_{\zeta(t) n}^{T}(t)\right)^{T}=\left(U_{\zeta(t)}^{T} \otimes I_{r}\right) e(t)
\end{array}\right.
$$

Then, (17) can be transformed as

$$
\begin{aligned}
\dot{V}(t) \leq & 2 \varphi_{\zeta(t)}^{T}(t)\left(I^{\prime}{ }_{n} \otimes P_{1} A+L^{\prime} \otimes P_{1} B K\right) \varphi_{\zeta(t)}(t) \\
& +2 \varphi_{\zeta(t)}^{T}(t)\left(L^{\prime} \otimes P_{1} B K\right) \chi_{\zeta(t)}(t) \\
& +\varphi_{\zeta(t)}^{T}(t)\left(I^{\prime}{ }_{n} \otimes\left(\mu P_{1} P_{1}+\mu I\right)\right) \varphi_{\zeta(t)}(t) \\
& +2 \varphi_{\zeta(t)}^{T}(t)\left(I^{\prime}{ }_{n} \otimes P_{1} D\right) \sigma_{\zeta(t)}(t) \\
& +\chi_{\zeta(t)}^{T}(t)\left(I_{n} \otimes\left(P_{2} A+A^{T} P_{2}+Q_{2}+Q_{2}^{T}\right)\right) \chi_{\zeta(t)}(t) \\
& +\chi_{\zeta(t)}^{T}(t)\left(I_{n} \otimes\left(\mu I+\mu P_{2}^{2}\right)\right) \chi_{\zeta(t)}(t)
\end{aligned}
$$

\section{Define}

$$
\begin{aligned}
\mathrm{Z}_{\zeta(t)}(t) & =\left(\mathrm{Z}_{\zeta(t) 1}^{T}(t), \ldots, \mathrm{Z}_{\zeta(t) n-1}^{T}(t)\right)^{T} \\
& =\left(\Pi_{1_{\zeta}(t)}^{T} \otimes I_{r-1}\right) \varphi_{\zeta(t)}(t) \\
W_{\zeta(t)}(t) & =\left(W_{\zeta(t) 1}^{T}(t), \ldots, W_{\zeta(t) n-1}^{T}(t)\right)^{T} \\
& =\left(\Pi_{1_{\zeta}(t)}^{T} \otimes I_{r-1}\right) \sigma_{\zeta(t)}(t) \\
\mathrm{E}_{\zeta(t)}(t) & =\left(\mathrm{E}_{\zeta(t) 1}^{T}(t), \ldots, \mathrm{E}_{\zeta(t) n}^{T}(t)\right)^{T} \\
& =\left(\Pi_{1_{\zeta}(t)}^{T} \otimes I_{r}\right) \chi_{\zeta(t)}(t)
\end{aligned}
$$

in which $\Pi_{1_{\zeta}(t)} \in R^{(n-1) \times(n-1)}$ and $\Pi_{\zeta(t)} \in R^{n \times n}$ are orthogonal matrices. Eq.(18) can be further formed as

$\dot{V}(t) \leq 2 \mathrm{Z}_{\zeta(t)}^{T}(t)\left(I_{n-1} \otimes P_{1} A+\Lambda_{1} \otimes P_{1} B K\right) \mathrm{Z}_{\zeta(t)}(t)$

$+2 Z_{\zeta(t)}^{T}(t)\left(\Lambda_{1} \otimes P_{1} B K\right) \mathrm{E}_{\zeta(t)}(t)$

$+\mathrm{Z}_{\zeta(t)}^{T}(t)\left(I_{n-1} \otimes\left(\mu P_{1}^{2}+\mu I\right)\right) \mathrm{Z}_{\zeta(t)}(t)$

$+2 Z_{\zeta(t)}^{T}(t)\left(I_{n-1} \otimes P_{1} D\right) W_{\zeta(t)}(t)$

$+\mathrm{E}_{\zeta(t)}^{T}(t)\left(I_{n} \otimes\left(P_{2} A+A^{T} P_{2}+Q_{2}+Q_{2}^{T}\right)\right) \mathrm{E}_{\zeta(t)}(t)$

$+\mathrm{E}_{\zeta(t)}^{T}(t)\left(I_{n} \otimes\left(\mu I+\mu P_{2}^{2}\right)\right) \mathrm{E}_{\zeta(t)}(t)$

The function is considered as follows for any $T>0$

$J_{T}=\int_{0}^{T} \mathrm{Z}^{T}(t) \mathrm{Z}(t) \mathrm{d} t-\gamma^{2} \int_{0}^{T} W^{T}(t) W(t) \mathrm{d} t$

With $V(0)=0$, we get

$J_{T}=\int_{0}^{T} \mathrm{Z}^{T}(t) \mathrm{Z}(t)-\gamma^{2} W^{T}(t) W(t)+\dot{V}(t) \mathrm{d} t-V(T)$

Set

$\phi_{\zeta(t) i}(t)=\left(\mathrm{Z}_{\zeta(t) i}^{T}(t), W_{\zeta(t) i}^{T}(t), \mathrm{E}_{\zeta(t) i}^{T}(t)\right)^{T}$

and combining (19) and (20), we can get

$$
\begin{aligned}
J_{T} \leq & \int_{0}^{T}\left(\sum_{i=1}^{n-1} \phi_{\zeta(t) i}^{T}(t) \Theta \phi_{\zeta(t) i}(t)+\mathrm{E}_{\zeta(t) n}^{T}\left(M+\mu P_{2}^{2}\right) \mathrm{E}_{\zeta(t) n}\right) \mathrm{d} t \\
& -V(T)
\end{aligned}
$$

in which

$$
\Theta=\left[\begin{array}{ccc}
\Omega+\mu P_{1}^{2} & P_{1} D & \lambda_{\varsigma(t) i} Q_{1} \\
* & -\gamma^{2} I & 0 \\
* & * & M+\mu P_{2}^{2}
\end{array}\right]
$$

Utilizing Schur Lemma [34], inequality (12) can be equivalently transformed as $\Theta<0$ and $M+\mu P_{2}^{2}<0$. Hence we 
obtain $J_{T}<0$, and the consensus objective as in Definition 1 is completed.

Remark 1 Limited to switching frequency and action delay, it is difficult to realize the slidingmode strategy (5) sometimes, and it may further cause chattering phenomenon. It is feasible to modify (5) to (21). In this situation, the error trajectories do not slide perfectly to zero, but within a small boundary layer around zero [35], the nonlinear input of the sliding-mode observers (3) can be designed as

$v_{i}(t)=\left\{\begin{array}{cc}-\rho \frac{F \Psi_{i}(t)}{\left\|F \Psi_{i}(t)\right\|+\delta}, & \Psi_{i}(t) \neq 0 \\ 0, & \Psi_{i}(t)=0\end{array}\right.$

where $\delta>0$ is a small constant.

Remark 2 During calculating $G, F$ and controller gain matrix $K$, because the matrices $C$ and $B$ are not square matrices actually, we can not directly obtain their inverse matrices. Therefore, utilizing the pseudo inverse technology for the matrices, we can get the observer gain matrix $G$, the controller gain matrix $K$ and $F$ in the following:

$$
\begin{aligned}
& G=P_{2}^{-1} Q_{2} C^{T}\left(C C^{T}\right)^{-1} \\
& K=\left(B^{T} B\right)^{-1} B^{T} P_{1}^{-1} Q_{1} \\
& F=\left(P_{2} D\right)^{T} C^{T}\left(C C^{T}\right)^{-1}
\end{aligned}
$$

Remark 3 By solving the feasible solution of LMI (12), the sliding-mode observer (3) reduces parameters' constraints in the design process. Furthermore, the consensus controller (8) based on the designed observer (3) could complete the state synchronization of MAS (1) with considering the unavailable states, nonlinear dynamics and external disturbances, simultaneously.

\section{Simulation example}

\section{Parameters calculation for multiple simple-pendulum systems}

In this section, we consider multiple simple-pendulum systems described in MASs (1) with four simple-pendulums [36], and the simple-pendulum system is shown in Fig. 2. For the $i$ th simple-pendulum, set $x_{i 1}=\theta_{i}, x_{i 2}=\dot{\theta}_{i}, x_{i 3}=i_{a i}$ as angular displacement, angular velocity, and armature current, respectively, and denote $x_{i}=\left[x_{i 1}^{T}, x_{i 2}^{T}, x_{i 3}^{T}\right]$. Then we obtain the dynamics of the $i t h$ simple-pendulum as

$\dot{x}_{i}(t)=A \dot{x}_{i}(t)+B u_{i}(t)+f\left(x_{i}(t)\right)+D \omega_{i}(t)$

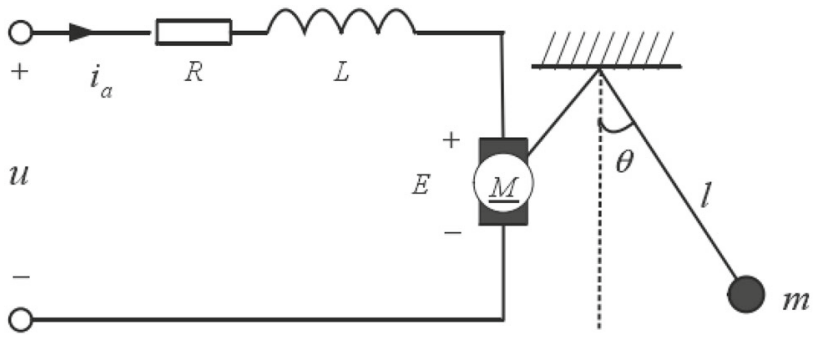

Fig. 2 Simple-pendulum system driven by DC motor

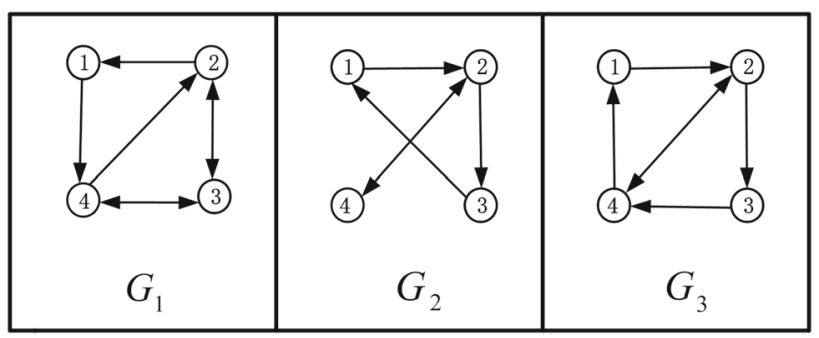

Fig. 3 Interaction graphs

where

$$
\begin{aligned}
\mathbf{A} & =\left(\begin{array}{ccc}
0 & 1 & 0 \\
0 & 0 & \frac{K_{T}}{m l^{2}} \\
0 & -\frac{K_{E}}{L} & -\frac{R}{L}
\end{array}\right), \mathbf{B}=\left(\begin{array}{c}
0 \\
0 \\
\frac{1}{L}
\end{array}\right) \\
f\left(x_{i}(t)\right) & =\left[0,-\frac{g}{l} \sin x_{i 1}(t), 0\right]^{T}
\end{aligned}
$$

$u$ represents the terminal voltage, $R$ and $L$ the resistance and inductance of the armature circuit respectively, $i_{a}$ and $E$ the armature current and the back electromotive force (EMF) of DC motor. $m$ and $l$ denote as the mass of the pendulum ball and the length of the pendulum rod, $K_{T}$ and $K_{E}$ represent torque constant and back EMF constant respectively.

The following system parameters are

$$
\begin{aligned}
& A=\left(\begin{array}{ccc}
0 & 1 & 0 \\
0 & 0 & 0.8 \\
0 & -0.15 & -1
\end{array}\right), B=\left(\begin{array}{l}
0 \\
0 \\
1
\end{array}\right), C=\left[\begin{array}{lll}
1 & 0 & 0 \\
0 & 0 & 1
\end{array}\right] \\
& D=\left[\begin{array}{lll}
0 & 1 & 1
\end{array}\right]^{T}, f\left(x_{i}(t)\right)=\left[\begin{array}{ll}
0-0.5 \sin x_{i 1}(t) & 0
\end{array}\right]^{T}
\end{aligned}
$$

and the external disturbance is supposed as $d_{i}=0.02 \cos t$.

The switching communication topologies are considered as the set $G_{1}, G_{2}, G_{3}$ in Fig. 3 with $\varsigma(t)=1,2,3$ which is switched every $\tau=1$ to the next topology as $G_{1} \rightarrow G_{2} \rightarrow$ $G_{3} \rightarrow G_{1} \rightarrow \cdots$ according to Fig. 4 .

Set $\mu=0.55, \gamma=0.55, \rho=1, \delta=0.01$. By solving asymptotical stability condition (12), the observer gain and 


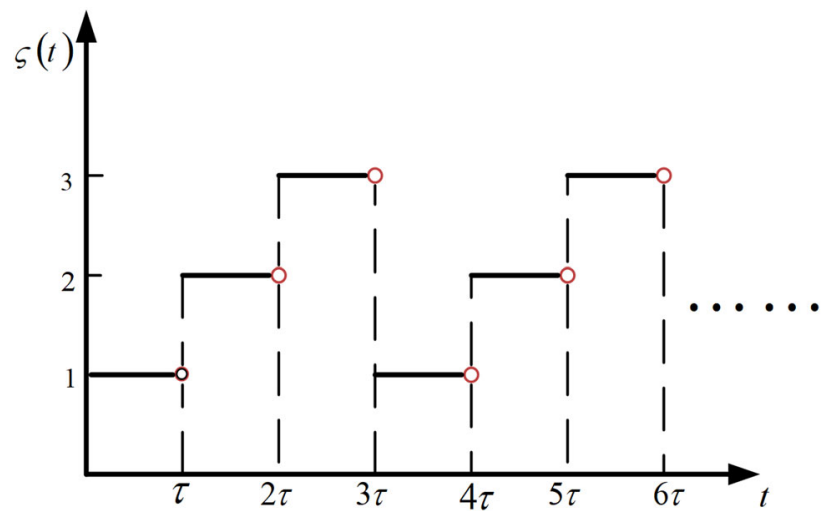

Fig. 4 Switching signal $\varsigma(t)$

controller gain matrices are obtained as

$$
\begin{aligned}
& G=\left[\begin{array}{cc}
-1.6991 & 0 \\
-0.7219 & 0.8098 \\
0 & -0.6991
\end{array}\right] \\
& K=[-0.3036-1.5322-1.2078]
\end{aligned}
$$

and other related parameters are computed as

$$
\begin{aligned}
P_{1} & =\left[\begin{array}{ccc}
0.4683 & -0.1642 & 0.0659 \\
-0.1642 & 0.4036 & -0.3002 \\
0.0659 & -0.3002 & 0.5940
\end{array}\right] \\
P_{2} & =\left[\begin{array}{ccc}
1.3813 & 0 & 0 \\
0 & 1.3813 & 0 \\
0 & 0 & 1.3813
\end{array}\right] \\
Q_{1} & =\left[\begin{array}{ccc}
-0.6302 & -0.0614 & 0.0282 \\
-0.0632 & -0.5808 & -0.0744 \\
0.0286 & -0.0761 & -0.3827
\end{array}\right] \\
Q_{2} & =\left[\begin{array}{ccc}
-2.3470 & -0.3841 & 0 \\
-0.9972 & -2.3470 & 1.1186 \\
0 & -2.0165 & -0.9657
\end{array}\right] \\
F & =\left[\begin{array}{ll}
0 & 1.3813
\end{array}\right]
\end{aligned}
$$

\section{Simulation analysis}

Due to the angular velocity of simple-pendulum systems cannot be measured actually, the designed observer (3) is mainly used to estimate it. In Fig. $4, e_{\mathrm{i} 2}(i=1,2,3,4)$ represents angular velocity error of four simple-pendulum systems, and we can see that $e_{\mathrm{i} 2}(i=1,2,3,4)$ converges to zero asymptotically, which concludes the designed slidingmode observer (3) can estimate the agents' states effectively.

Furthermore, the angular velocity of four simple-pendulum systems is also estimated by the observer designed in [19]. The nonlinearity and disturbance are considered in 8-25 seconds and Fig. 6 displays the angular velocity error of the

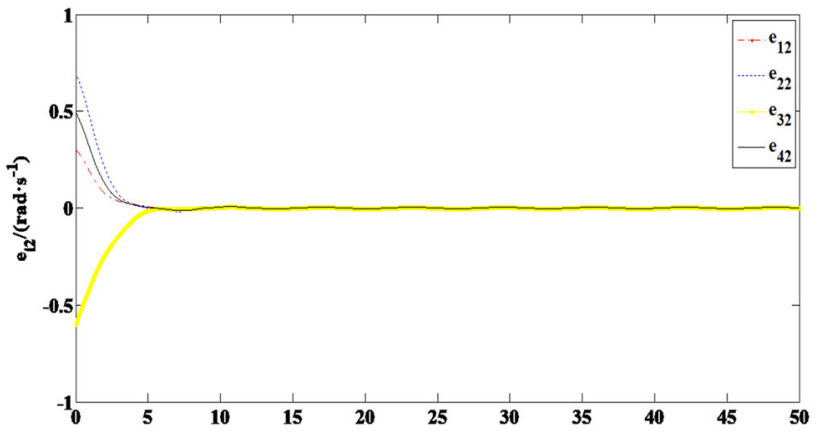

Fig. 5 State errors of the angular velocity in this work

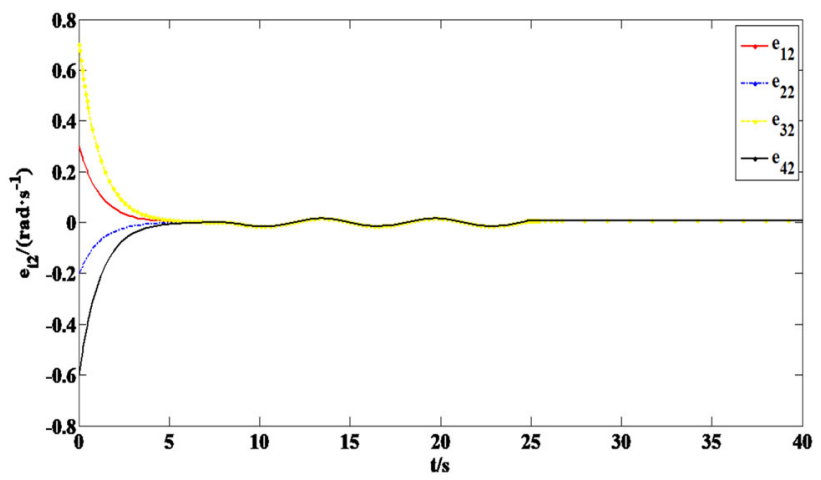

Fig. 6 State errors of the angular velocity in [19]

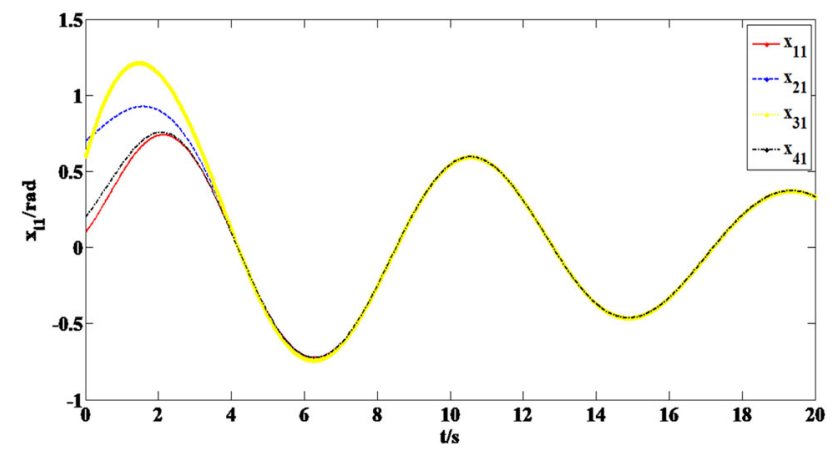

Fig. 7 Angular displacement under the controller (8)

observer in [19]. Comparing Figs. 5 and 6, the state error curves in Fig. 6 show obvious fluctuation in 8-25 seconds, and the observer designed in our work possesses the superior in dealing with disturbances and nonlinearity.

Under the designed control protocol (8) with distributed observers (3), Figs. 7, 8, 9 demonstrate respectively angular displacement, angular velocity and armature current curves of four simple pendulums could achieve the goal of consensus control finally. By observing the simulation results on designed sliding-mode observers (3) and distributed controller (8), we can summarize that the proposed distributed control protocol based on sliding-mode observers is effective and asymptotically stable for nonlinear multi-agent systems with disturbances in our work. 


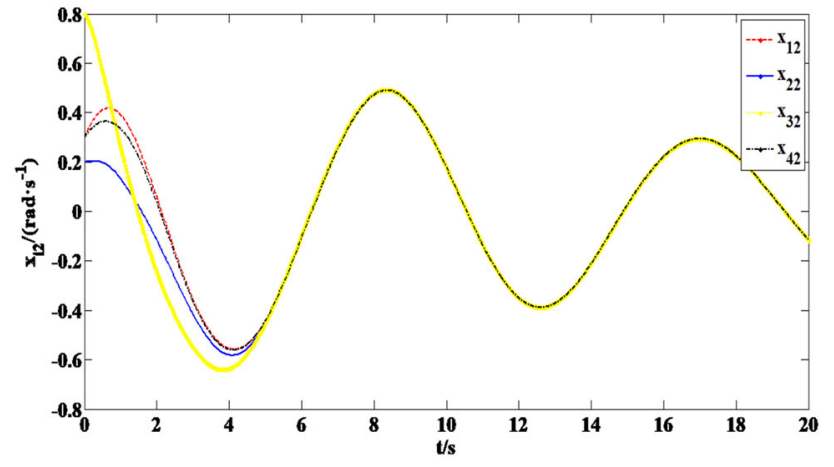

Fig. 8 Angular velocity under the controller (8)

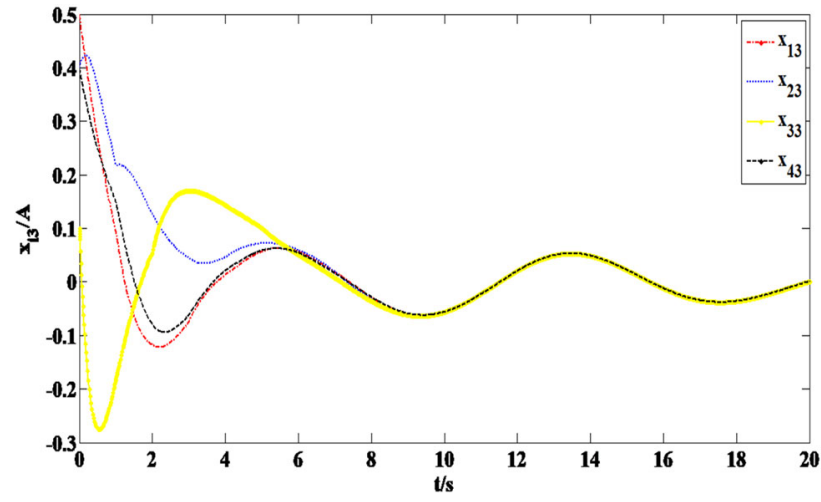

Fig. 9 Armature current under the controller (8)

\section{Conclusions}

This work has solved distributed consensus control for a class of MASs considering unavailable states, nonlinearity and unknown disturbances under switching topologies. The distributed sliding-mode observer has been proposed to estimate states of nonlinear MASs with external disturbances. Then, the consensus control law has been designed utilizing estimated state variables. The condition of robust consensus has been obtained satisfying the anti-jamming performance index. The simulation results about multiple simple-pendulum systems further illustrate that our proposed observer and controller are valid. Future work will be deserved on distributed cooperative control in higher-order nonlinear systems with unmeasurable states, external disturbances and communication delays under jointly connected topologies.

Acknowledgements This work was supported by the Natural Science Foundation of China (61503045), the key science and technology projects of Jilin province (20200401075GX).

Open Access This article is licensed under a Creative Commons Attribution 4.0 International License, which permits use, sharing, adaptation, distribution and reproduction in any medium or format, as long as you give appropriate credit to the original author(s) and the source, provide a link to the Creative Commons licence, and indi- cate if changes were made. The images or other third party material in this article are included in the article's Creative Commons licence, unless indicated otherwise in a credit line to the material. If material is not included in the article's Creative Commons licence and your intended use is not permitted by statutory regulation or exceeds the permitted use, you will need to obtain permission directly from the copyright holder. To view a copy of this licence, visit http://creativecomm ons.org/licenses/by/4.0/.

\section{References}

1. Arokiasami WA, Vadakkepat P, Tan KC, Srinivasan D (2016) Interoperable multi-agent framework for unmanned aerial/ground vehicles: towards robot autonomy. Complex Intell Syst 2(1):45-59

2. Fax J, Murray RM (2004) Information flow and cooperative control of vehicle formations. IEEE Trans Autom Control 49(9):14651476

3. Chang I, Park SY, Choi KH (2009) Decentralized coordinated attitude control for satellite formation flying via the state-dependent Riccati equation technique. Int J NonLinear Mech 44(8):891-904

4. Yu JZ, Wang C, Xie GM (2016) Coordination of multiple robotic fish with applications to underwater robot competition. IEEE Trans Ind Electron 62(2):1280-1288

5. Dong XW, Yan Z, Zhang R (2017) Time-varying formation tracking for second-order multi-agent systems subjected to switching topologies With application to quadrotor formation flying. IEEE Trans Ind Electron 64(4):5014-5024

6. Li JG, Deng GQ, Luo CW et al (2016) A hybrid path planning method in unmanned air/ground vehicle (UAV/UGV) cooperative systems. IEEE Trans Veh Technol 65(12):9585-9596

7. Wen GH, Duan ZS, Ren W, Chen GR (2014) Distributed consensus of multi-agent systems with general linear node dynamics and intermittent communications. Int J Robust Nonlinear Control 24(16):2438-2457

8. Li ZK, Liu XD, Lin P, Ren W (2011) Consensus of linear multiagent systems with reduced-order observer-based protocols. Syst Control Lett 60(7):510-516

9. Wang H, Yu WW, Ding ZT, Yu XH (2019) Tracking consensus of general nonlinear multi-agent systems with external disturbances under directed networks. IEEE Trans Autom Control: 1-1

10. Liang HJ, Zhou Y, Ma H, Zhou Q (2019) Adaptive distributed observer approach for cooperative containment control of nonidentical networks. IEEE Trans Syst Man Cybern Syst 49(2):299-307

11. Zou WC, Shi P, Xiang ZR, Shi Y (2020) Finite-time consensus of second-order switched nonlinear multi-agent systems. IEEE Trans Neural Netw Learn Syst 31(5):1757-1762

12. Liu J, Zhang YL, Yu Y, Sun CY (2019) Fixed-time eventtriggered consensus for nonlinear multiagent systems without continuous communications. IEEE Trans on Syst Man Cybern Syst 49(11):2221-2229

13. Liu J, Zhang YL, Yu Y, Sun CY (2020) Fixed-time leader-follower consensus of networked nonlinear systems via event/self-triggered control. IEEE Trans Neural Netw Learn Syst 31(11):5029-5037

14. Liu J, Yu Y, He HB, Sun CY (2020) Team-triggered practical fixed-time consensus of double-integrator agents with uncertain disturbance. IEEE Trans Cybern: 1-10

15. Zhang HG, Cai YL, Wang YC, Su HG (2020) Adaptive bipartite event-triggered output consensus of heterogeneous linear multiagent systems under fixed and switching topologies. IEEE Trans Neural Netw Learn Syst 31(11):4816-4830

16. Wang YC, Li HF, Qiu XJ, Xie XP (2020) Consensus tracking for nonlinear multi-agent systems with unknown disturbance by using model free adaptive iterative learning control. Appl Math Comput $365: 124701$ 
17. Mao J, Karimi HR, Xiang ZR (2019) Observer-based adaptive consensus for a class of nonlinear multiagent systems. IEEE Trans Syst, Man, Cybern: Syst 49(9):1893-1900

18. Xu BB, Li JW, Gao LX, Zhang H (2015) Distributed reduced-order observer-based approach to consensus problems for linear multiagent systems. IET Control Theory Appl 9(5):784-792

19. Yang TT, Zhang PF, Yu SH (2017) Consensus of linear multi-agent systems via reduced-order observer. Neurocomputing 240:200 208

20. Jiang YL, Wang HQ, Wang SQ (2019) Distributed $H_{\infty}$ consensus control for nonlinear multi-agent systems under switching topologies via relative output feedback. Neural Comput Appl 31:1-9

21. Xue LR, Liu ZG, Sun ZY et al (2019) New results on robust tracking control for a class of high-order nonlinear time-delay systems. Int J Syst Sci 50(10):2002-2014

22. Liang HJ, Zhang YH, Huang TW, Ma H (2019) Prescribed performance cooperative control for multi-agent systems with input quantization. IEEE Trans Cybern: 1-10

23. Wang XH, Xiang ZR (2019) Global finite-time stabilisation of high-order nonlinear systems: a dynamic gain-based approach. Int J Syst Sci 50(5-8):1677-1687

24. Wang YC, Zheng LF, Zhang HG, Zheng WX (2020) Fuzzy observer-based repetitive tracking control for nonlinear systems. IEEE Trans Fuzzy Syst 28(10):2401-2415

25. Lee DJ, Park YJ, Park YS (2012) Robust sliding mode descriptor observer for fault and output disturbance estimation of uncertain systems. IEEE Trans Autom Control 57(11):2928-2934

26. Raoufi R, Marquez HJ, Zinober ASI (2010) $H_{\infty}$ sliding mode observers for uncertain nonlinear Lipschitz systems with fault estimation synthesis. Int J Robust Nonlinear Control 20(16):17851801

27. Shen QK, Shi P, Shi Y (2016) Distributed adaptive fuzzy control for nonlinear multi-agent systems via sliding mode observers. IEEE Trans Cybern: $1-12$

28. Li XL, Luo XY, Li SB, Li JJ, Guan XP (2017) Consensus of secondorder nonlinear multi-agent systems via sliding mode observer and controller. J Syst Eng Electron 28:756-765
29. Wang XY, Li SH, Lam J (2016) Distributed active anti-disturbance output consensus algorithms for higher-order multi-agent systems with mismatched disturbances. Automatica 74:30-37

30. Mondal S, Rong S, Xie LH (2017) Heterogeneous consensus of higher-order multi-agent systems with mismatched uncertainties using sliding mode control. Int J Robust Nonlinear Control 27(13):2303-2320

31. Zhu YZ, Zheng WX (2020) Observer-based control for cyberphysical systems with doS attacks via a cyclic switching strategy. IEEE Trans Autom Control 65(8):3714-3721

32. Jiang YL, Liu JC, Wang SQ (2014) Robust integral sliding-mode consensus tracking for multi-agent systems with time varying delay. Asian J Control 18(1):224-235

33. Lin P, Jia YM, Li L (2008) Distributed robust $H_{\infty}$ consensus control in directed networks of agents with time-delay. Syst Control Lett 57(8):643-653

34. Yaz EE (1998) Linear matrix inequalities in system and control theory. Proc IEEE 86(12):2473-2474

35. Tan CP, Edwards C (2003) Sliding mode observers for robust detection and reconstruction of actuator and sensor faults. Int J Robust Nonlinear Control 13(5):443-463

36. Jiang YL, Wang SQ, Li YC, Liu DR (2016) Distributed consensus tracking control for multiple simple-pendulum network systems. In: Proceeding of the35th Chinese Control Conference(CCC), Chengdu, China, pp. 7556-7560

Publisher's Note Springer Nature remains neutral with regard to jurisdictional claims in published maps and institutional affiliations. 